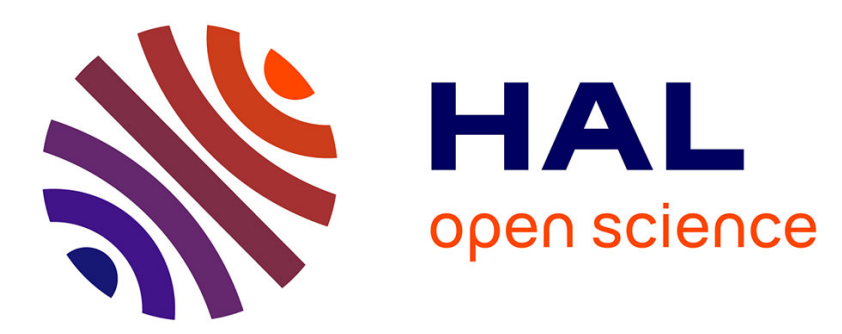

\title{
Optimal trajectory determination and tracking of an autoclave curing process: A model based approach
}

Pascal Dufour, Youssoufi Touré, Dennis J Michaud, Prasad S. Dhurjati

\section{To cite this version:}

Pascal Dufour, Youssoufi Touré, Dennis J Michaud, Prasad S. Dhurjati. Optimal trajectory determination and tracking of an autoclave curing process: A model based approach. European Control Conference (ECC), Sep 1999, Karlsruhe, Germany. Paper F1033-6. hal-00353148v2

\section{HAL Id: hal-00353148 \\ https://hal.science/hal-00353148v2}

Submitted on 22 Jan 2009

HAL is a multi-disciplinary open access archive for the deposit and dissemination of scientific research documents, whether they are published or not. The documents may come from teaching and research institutions in France or abroad, or from public or private research centers.
L'archive ouverte pluridisciplinaire HAL, est destinée au dépôt et à la diffusion de documents scientifiques de niveau recherche, publiés ou non, émanant des établissements d'enseignement et de recherche français ou étrangers, des laboratoires publics ou privés. 
This document must be cited according to its final version which is published in a conference proceeding as:

P. Dufour ${ }^{1}$, Y. Touré ${ }^{1}$, D.J. Michaud ${ }^{2}$, P.S. Dhurjati ${ }^{2}$, "Optimal trajectory determination and tracking of an autoclave curing process: A model based approach", Proceedings of the $5^{\text {th }}$ European Control Conference (ECC), Paper F1033-6,

Karlsruhe, Germany, august 31-september 3, 1999.

All open archive documents of Pascal Dufour are available at: http://hal.archives-ouvertes.fr/DUFOUR-PASCAL-C-3926-2008

The professional web page (Fr/En) of Pascal Dufour is: http://www.lagep.univ-lyon1.fr/signatures/dufour.pascal

1

Université de Lyon, Lyon, F-69003, France; Université Lyon 1;

CNRS UMR 5007 LAGEP (Laboratoire d'Automatique et de GEnie des Procédés),

43 bd du 11 novembre, 69100 Villeurbanne, France

Tel +33(0) 472431845 - Fax +33 (0) 472431699

http://www-lagep.univ-lyon1.fr/ http://www.univ-lyon1.fr http://www.cnrs.fr

2

University of Delaware,

Department of Chemical Engineering,

Newark, DE 19716, USA

http://www.udel.edu 


\title{
OPTIMAL TRAJECTORY DETERMINATION AND TRACKING OF AN AUTOCLAVE CURING PROCESS: A MODEL BASED APPROACH
}

\author{
P. Dufour ${ }^{1}$, Y. Touré ${ }^{2}$, D.J. Michaud ${ }^{3}$, P.S. Dhurjati ${ }^{3}$ \\ ${ }^{1}$ LAGEP UPRES-A CNRS Q 5007 \\ Université Claude Bernard \\ 43, bd du 11 Novembre 1918 \\ 69622 Villeurbanne cedex, France \\ ${ }^{2}$ IUT de Bourges \\ Université d'Orléans \\ 63 Avenue de Lattre de Tassigny \\ 18020 Bourges cedex, France \\ ${ }^{3}$ Department of Chemical Engineering and Center for Composite Materials \\ University of Delaware \\ Newark, DE 19716, U.S.A \\ Fax: ${ }^{1} 472431699,{ }^{2} 248238017,{ }^{3}$ (302) $831-1048$ \\ e-mail: ${ }^{1}$ dufour@www-lagep.cpe.fr, ${ }^{2}$ Youssoufi.Toure@bourges.univ-orleans.fr, \\ ${ }^{3}$ michaud,dhurjati@che.udel.edu
}

Keywords: Model predictive control, constrained optimization, distributed parameter system control, curing process.

\begin{abstract}
This work deals with a model-based approach to nonlinear (NL) parabolic distributed parameter system control. We address two problems in the optimization and control of the autoclave lay-up curing process as presented in [11]. The first is to find the best process trajectory corresponding to the shortest possible operating time. The second is to determine the process control required to track the optimized trajectory within the constraints of the system. In previous work, a simulation based approach was used called local criteria optimization (LCO) [10]. Here the former problem is stated as a constrained optimization problem. A predictive type approach with a penalty method is solved by the LevenbergMarquardt (LM) algorithm. For the tracking problem, the well-known Internal Model Control (IMC) structure is used for the predictive control strategy. Finally, a timevarying linear model around an initial profile with parameters depending on the nonlinear model is used for the on-line optimization like in [4].
\end{abstract}

\section{Introduction}

This paper deals with the distributed parameter systems (DPS) control governed by non-linear parabolic partial differential equations where we state two problems. Find the control and output trajectories such that the process output reaches a minimum extent in a minimum time. Then, find the process control in order to track in process output the best trajectory induced by the first problem. Concerning the former problem, a first approach consists in the model based approach. The most direct way is using the Pontryagin's minimum principle developed through calculus of variations. But, in spite of basic methodology that guarantees optimal solutions, it does not seem to be easily implementable for NL DPS [8]. It is also possible to use an expert control [7] where finding pseudo-rules is a very difficult task. For batch processes, a simulation based optimization scheme with heuristic rules for control has been found to be more successful. This approach was previously applied to the process described in [10].

In this work, we propose a model-based approach. A constrained optimization problem, set in finite dimension, is combined with a predictive approach and solved using a nonlinear programming (NLP).

Few works have dealt with distributed parameter systems. The existed ones deal with structurally interested cases for 
one partial derivative equation but not for a set of equations. A first approach consists of using a transformation method based on the inverse scattering problem developed by Magri [9], where an exact linearization by geometrical transformation is proposed. The main problem is that the order of this transformation has to be fixed by a non trivial manner. In the nonlinear distributed parameter systems study, the most popular approach is the linearization approach, or else the finite dimensional approximation [12]. Concerning the control synthesis, explicit control law becomes more difficult: in [1], the problem is to solve Riccati equations, but therefore, it is still an open loop control. In [6], a structural approximation of the DPS is done in finite dimension taking into account of constraints. In this paper, we use an optimization approach taking into account of various constraints. We use also the Internal Model Control structure in its indirect version that brings to a predictive control approach by minimizing a criterion over a receding horizon [3, 4].

This approach is developed through an application example: an autoclave curing process used to manufacture composites from a thermosetting polymer matrix. The first objective is to find the fastest curing cycle, taking into account of constraints on the temperature and the degree of cure. Then, the control aim is to track a given temperature profile in process output. In the first section that follows, we present the autoclave curing process and its control problem: we describe the nonlinear boundary control system. In the next section, the reference determination problem is stated as a constrained optimization and the results are shown. The tracking problem, stated also in term of constrained optimization, is then exposed.

\section{Process and control objectives}

The process being studied is an autoclave curing process used to manufacture composites with a thermosetting polymer matrix. Prior to cure, the polymer is a viscous fluid. The assembly (Fig. 1) is sealed in a vacuum-bag and placed in an autoclave. It is then subjected to a prescribed autoclave temperature $T_{a}(t)$ (the process control variable) and pressure cycle, known as a cure cycle. Due to the application of heat, the resin solidifies through an irreversible exothermic chemical reaction of cure. The model version used here is in one spatial dimension since a 2-D problem has demonstrated that the temperature gradients in the lateral direction were negligible. The critical dimension is therefore across the thickness and is divided in three parts $\mathcal{D}_{u}, \mathcal{D}_{c}, \mathcal{D}_{d}$ (Fig. 2).

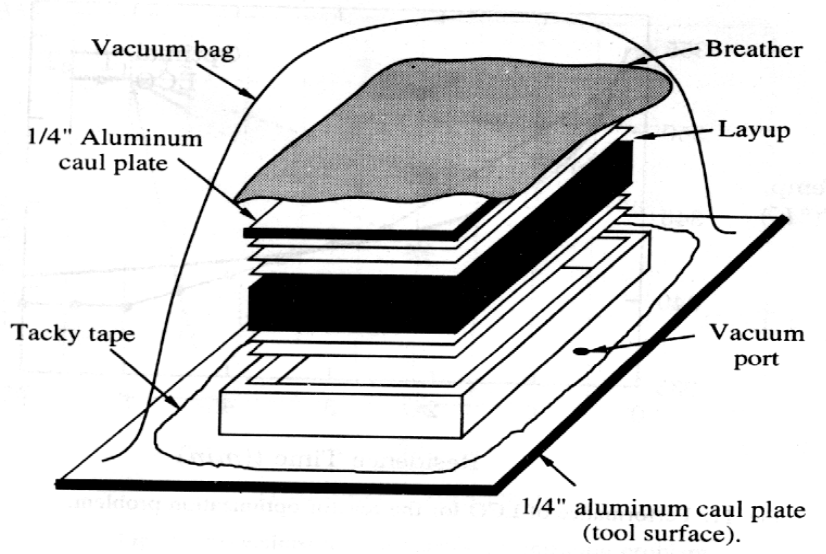

Figure 1: Composite layup [10].

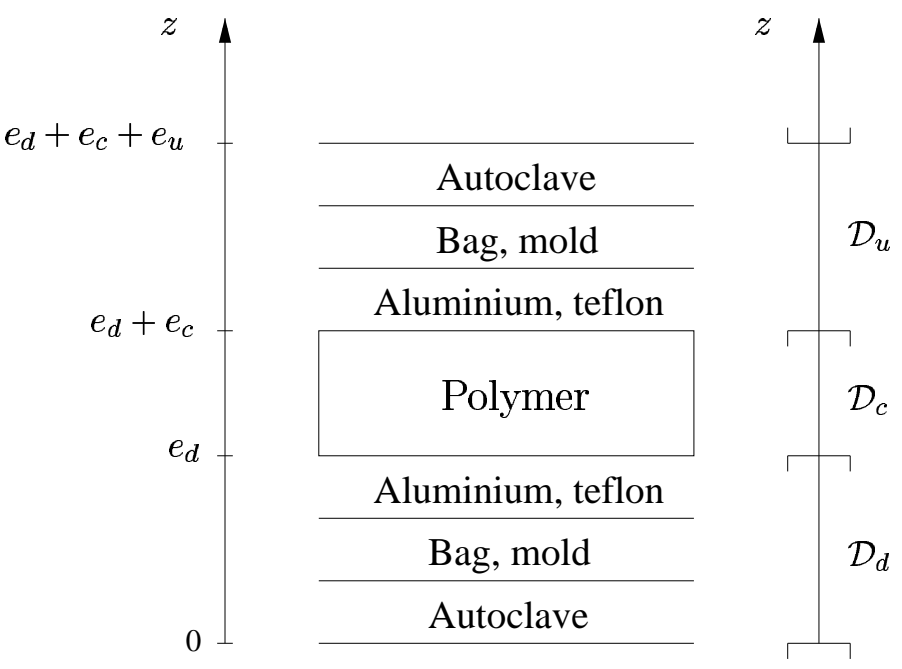

Figure 2: Spatial domains.

At the edge of the composite $\left(\mathcal{D}_{u}, \mathcal{D}_{d}\right)$, heat transfer resistance in the autoclave, the bag, the fabric and the mold have been lumped. In the composite part $\left(\mathcal{D}_{c}\right)$, the behaviour of temperature $T(z, t)$ is linked with the distributed degree of cure $\alpha(z, t)$ via the internal heat generation term. This degree of cure is equivalent to an extent of reaction and ranges from 0 to 1 .

\subsection{Nonlinear boundary control system}

The curing process can then be described by the following NL system $\left(\mathcal{S}_{N L}\right)$ [10] (where the model parameters description can be found): 


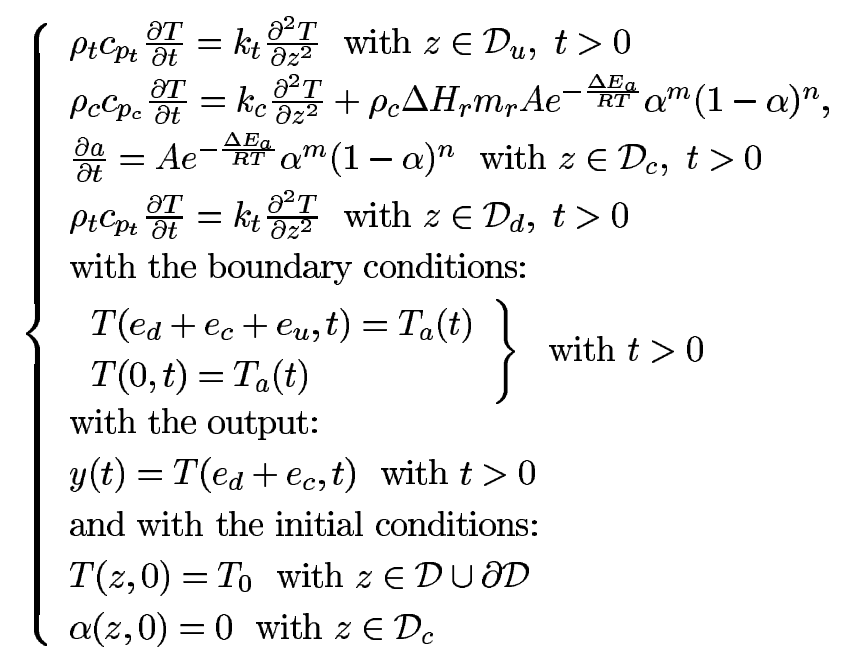

where the center point is located at $c=\frac{e_{d}+e_{c}+e_{u}}{2}$, $\partial \mathcal{D}=\left\{0, e_{d}+e_{c}+e_{u}\right\}$ and $\mathcal{D}=\mathcal{D}_{d} \cup \mathcal{D}_{c} \cup \mathcal{D}_{u}$. This system can be represented by a more general boundary control nonlinear distributed parameter system $\left[\left(\mathcal{S}_{N L}\right), \bar{\omega}(z), \omega(z, t), u(t), y(t), A, B, C, z \in \mathcal{D} \cup \partial \mathcal{D}, t>\right.$ $0]$ :

$$
\left(\mathcal{S}_{N L}\right)\left\{\begin{array}{l}
\frac{\partial w}{\partial t}(z, t)=A(\omega(z, t)) \text { with } z \in \mathcal{D}, t>0 \\
B(\omega(z, t), u(t))=0 \text { with } z \in \partial \mathcal{D}, t>0 \\
y(t)=C \omega(z, t) \text { with } z \in \mathcal{D} \cup \partial \mathcal{D}, t>0 \\
\omega(z, 0)=\bar{\omega}(z)
\end{array}\right.
$$

where $\omega(z, t)$ is the state defined on a Hilbert space $X, u(t)$ is the control and $y(t)$ the output. $A$ is a NL operator, $B$ and $C$ are bounded operators. Moreover, we assume that this NL model is regular in the sense of [2].

\section{Reference determination}

The manufacture of such thick composites is fraught of problems. The most detrimental effect is an internal temperature exotherm resulting from the heat generated by the cure reaction within the thermosetting resin. Hence, large thermal gradients can develop, which result in nonuniform curing. Moreover, if the part cures from outside inward, the large thermal stresses trapped in the part can lead to delamination and render it unusable. The task in optimizing lies therefore in decreasing the process time, while simultaneously improving the reliability and quality of the final product.

The problem is to find the control variable $u(t)$ such that the operating time is minimum taking into account of the following constraints: limit thermal gradients and ensure an inside-out curing. Obviously, the determinated control variable must be physically applicable: its amplitude and its control must be bounded. This problem can be expressed as a discrete time constrained optimization problem. At each sampling time $k$, we seek a vector $\tilde{p}$ minimizing a performance index $J$. This vector $\tilde{p}$ is the $N_{c}$ horizon control sequence. At each sampling time $k$, only the first component of the final $\tilde{p}$ is kept and the procedure is performed again at the next sampling time. The performance index is chosen in order to maximize the degree of cure dynamic at the center of the polymer over a receding horizon $N_{p}$ :

$$
\left(\mathcal{P}_{0}\right)\left\{\begin{array}{l}
\max _{\tilde{p}} J(\tilde{p})=\frac{1}{2} \sum_{j=k+1}^{j=k+N_{p}}\|\dot{\alpha}(c, j)\|^{2} \\
\tilde{p}=\left(u(k) \ldots u\left(k+N_{c}-1\right)\right)^{T}
\end{array}\right.
$$

The nco constraints must be satisfied at any time:

$$
g_{i, \min } \leq g_{i}(w, u) \leq g_{i, \max } \quad(1 \leq i \leq n c o)
$$

Then, these constraints are reformulated by rescaling them such that they have the same weight in the problem:

$$
\left(\mathcal{C}_{0}\right)\left\{\begin{array}{l}
-\frac{g_{i}(w, u)-g_{i, \min }}{g_{i, \max }-g_{i, \min }} \leq 0 \\
\frac{g_{i}(w, u)-g_{i, \min }}{g_{i, \max }-g_{i, \min }}-1 \leq 0
\end{array}\right.
$$

The maximization problem $\left(\mathcal{P}_{0}\right)$ is finally formulated under an equivalent minimization problem:

$$
\left(\mathcal{P}_{c}\right)\left\{\begin{array}{l}
\min _{\tilde{p}} J(\tilde{p})=\frac{1}{2} \sum_{j=k+1}^{j=k+N_{p}} \frac{1}{\|\dot{\alpha}(c, j)\|^{2}} \\
c_{i}(\tilde{p}) \leq 0 \quad(i \in I=\{1 \rightarrow 2 n c o\}) \\
\tilde{p}=\left(u(k) \ldots u\left(k+N_{c}-1\right)\right)^{T}
\end{array}\right.
$$

In order to solve the constrained optimization problem $\left(\mathcal{P}_{c}\right)$, we adopt a nonlinear programming method that combines the interior and exterior penalty methods [5], a transformation method and an unconstrained nonlinear resolution method. The advantage is that constraints can be easily introduced in the formulation. We then expressed the constrained problem into an unconstrained penalized optimization problem where the new performance index $J_{\text {tot }}$ to minimize is:

$$
\left(\mathcal{P}_{u}\right)\left\{\begin{array}{l}
\min _{\tilde{p}} J_{t o t}(\tilde{p})=J(\tilde{p})+p_{\text {int }} J_{\text {int }}\left(c_{i}(\tilde{p})\right)+p_{\text {ext }} J_{\text {ext }}\left(c_{i}(\tilde{p})\right) \\
\tilde{p}=\left(u(k) \ldots u\left(k+N_{c}-1\right)\right)^{T}
\end{array}\right.
$$

From the previous performance index $J$ in $\left(\mathcal{P}_{c}\right)$, we have added an interior (resp. exterior) penalty function depending on the constraints. It is weighted by a penalty coefficient $p_{\text {int }}$ (resp. $p_{\text {ext }}$ ).

\subsection{Interior penalty method}

The main advantage of this method is that we can ensure that every constraint is checked at any time for any control sequence $\tilde{p}$ tried by the resolution method. It is therefore a very interesting way if we have to determine an on-line control: the algorithm has to be able to give a physical solution even if the resolution method is stopped 
due the maximal computational time constraint. The major known drawback is the initialization problem. For the nci constraints set here, the interior penalty function $J_{\text {int }}$ can be an inverse barrier function [5]:

$$
J_{i n t}\left(c_{i}(\tilde{p})\right)=\sum_{i=1}^{i=n c i} \frac{-1}{c_{i}(\tilde{p})}
$$

$J_{\text {int }}$ is defined on $\stackrel{\circ}{K}=\left\{\tilde{p}\right.$ such that $\left.c_{i}(\tilde{p})<0\right\}$. The advantage of this formulation is that, in the domain $\stackrel{\circ}{K}$, $J_{\text {int }}$ is always a penalty term since it takes always positive value.

\subsection{Exterior penalty method}

The advantage of this method is that the vector $\tilde{p}$ can be initialized anywhere. The induced drawback is that the constraints can be violated during the calculation time. For the nce constraints stated here, the exterior penalty function $J_{\text {ext }}$ is [5]:

$$
\left\{\begin{array}{l}
J_{e x t}\left(c_{i}(\tilde{p})\right)=\sum_{i=1}^{i=n c e} f\left(c_{i}(\tilde{p})\right) \\
f\left(c_{i}(\tilde{p})\right)=\max ^{2}\left(0, c_{i}(\tilde{p})\right)
\end{array}\right.
$$

\subsection{Resolution method}

The problem $\left(\mathcal{P}_{u}\right)$ can now be solved by any unconstrained optimization method. In this domain, the LevenbergMarquardt's algorithm is one of the most important [5]. The evolution in the parameters space $P$ follows the law :

$$
\tilde{p}^{i+1}=\tilde{p}^{i}-\left(\nabla^{2} J_{t o t}^{i}+\lambda I\right)^{-1} \nabla J_{t o t}^{i}
$$

\section{Algorithm modification}

But, a structural problem can occur. Indeed, this algorithm is able to find a solution anywhere in $P$. Since the interior penalty function $J_{\text {int }}$ is defined anywhere out of the boundary of the constraints domain $K$, some $\tilde{p}$ vector are prohibited. Moreover, we have to ensure that any $\tilde{p}$ tried by the algorithm is such that $J_{\text {int }}$ is a positive term otherwise it is not a penalty term. This leads to a modification in the algorithm by introducing a new parameter $\theta$ :

$$
\tilde{p}^{i+1}=\tilde{p}^{i}-\theta\left(\nabla^{2} J_{t o t}^{i}+\lambda I\right)^{-1} \nabla J_{t o t}^{i}
$$

\section{Parameter transformation}

Considering the nonlinear model $\left(\mathcal{S}_{N L}\right)$, a numerical divergence can happen with the Arrhenius kinetic behavior and the temperature therefore has to be kept over a minimal value. The only way to avoid a small polymer temperature is to forbid small control values. So, the constraints dealing with the control amplitude are stated using a parameter transformation instead of the interior penalty function :

$$
u_{\min } \leq u \leq u_{\max }
$$

becomes:

$$
u=\frac{u_{\max }+u_{\min }}{2}+\frac{u_{\max }-u_{\min }}{2} \tanh (w)
$$

where $\tilde{p}=\left(w(k) \ldots w\left(k+N_{c}-1\right)\right)^{T}$ is the new parameters vector to find that can take value anywhere in $P$. In this application, this replaces implicitly $\theta$.

\subsection{Simulation results}

Concerning the constraints, the control sequence computed must be physically attainable:

$$
\begin{aligned}
& T_{a_{\min }} \leq T_{a} \leq T_{a_{\max }} \\
& \dot{T}_{a_{\min }} \leq \dot{T}_{a} \leq \dot{T}_{a_{\min }}
\end{aligned}
$$

As we mentioned before, control amplitude constraints have been expressed using a transformation method. Since computational time is not constrained here, the other constraints are set using an exterior penalty function. We just take into account of the inside-out cure problem. This is done by introducing fictitious constraints. This means that we express our process behavior skill. At first sight, the inside-out cure happens if the temperature inside the polymer is almost uniform and has reached a minimum extent. We have therefore put thermal gradients constraints and extent of cure gradient constraints :

$$
\begin{aligned}
\Delta T_{\min } & \leq T\left(e_{d}+e_{c}, t\right)-T(c, t) \leq \Delta T_{\max } \\
\Delta \alpha_{\min } & \leq \alpha\left(e_{d}+e_{c}, t\right)-\alpha(c, t) \leq \Delta \alpha_{\max }
\end{aligned}
$$

The previous framework is then applied to the optimization problem $\left(\mathcal{P}_{u}\right)$. The remaining problem is to tune the horizons. With $N_{c}=1, N_{p}=15$, the best resulting trajectory for temperatures and extents of cure inside the composite are shown:

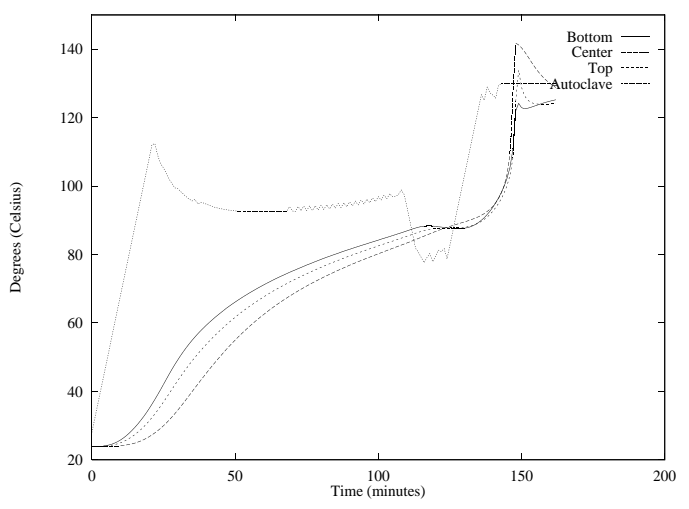

Figure 3: Temperatures 


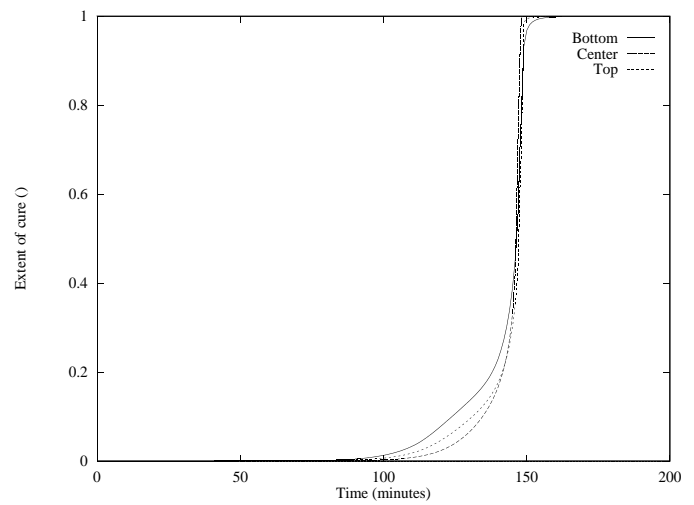

Figure 4: Through thickness curing behaviour

In this case, the cure is completed in 163 minutes. The inside-out cure is ensure but the thermal gradient seems to be too important when the exothermic reaction occurs. At each sampling time, the algorithm output condition ensures that the most violated exterior constraints related to the final solution $\tilde{p}$ does not exceed $1 \%$ of the constraints scale $\left(\operatorname{cf}\left(\mathcal{C}_{0}\right)\right)$ :

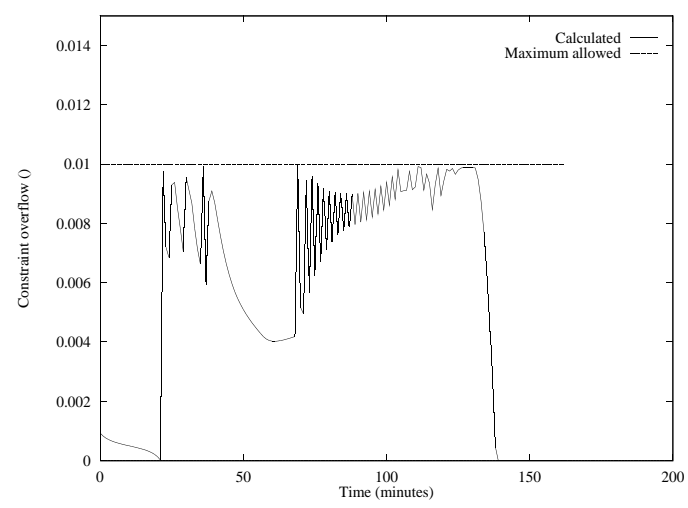

Figure 5: Algorithm output condition

With this method, we have found the best manner in which to cure the polymer. The problem is now to find the control of the closed-loop system such that the output temperature tracks effectively the optimal output temperature $y_{r e f}(t)$ induced we just have found. This is a more classical control problem that follows.

\section{Nonlinear predictive control}

The control problem can be stated as follows: for a given optimal output temperature trajectory (Fig. 3), find a control strategy such that the process output tracks this profile with the above control constraints and the extent of cure crossover constraint. We restate this control problem again as a constrained optimization problem with a predictive type approach in the Internal Model Control (IMC) like structure.

\subsection{IMC structure}

The objective is to find a controller such that the process output $y_{p}$ tracks some reference points set $y_{r e f}$ in spite of some modelling error and added disturbances. It can be an explicit control law [13]. But for nonlinear systems and time-varying linear systems, the indirect one seems to be more feasible: at each sampling time, a control sequence over a control horizon optimizing a criterion is computed [3]. It uses the process output predicted over the prediction horizon by the model. The control structure becomes the following one:

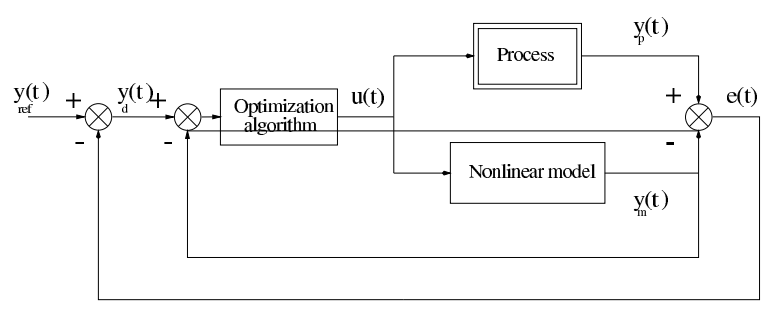

Figure 6: Internal Model Control structure

The optimization problem is similar to $\left(\mathcal{P}_{C}\right)$. It is formulated in the discrete form by minimizing a classical tracking criterion over a receding horizon $N_{p}$ :

$$
\left\{\begin{array}{l}
\max _{\tilde{p}}=\frac{1}{2} \Sigma_{j=k+1}^{j=k+N_{p}}\left\|y_{r e f}(j)-y_{p}(j)\right\|^{2} \\
\tilde{p}=\left(u(k) \ldots u\left(k+N_{c}-1\right)\right)^{T} \\
g_{i, \min } \leq g_{i}(w, u) \leq g_{i, \max }(1 \leq i \leq n c o)
\end{array}\right.
$$

To solve this constrained optimization problem, we choose again the penalty method associated with the LevenbergMarquardt method. The computation is made at each sampling time to get the optimal control sequence $\tilde{p}$, but only the first component $u(k)$ is applied to both model and process. As expected, it can be viewed as the dual problem of the optimal trajectory determination. Since we considered an off-line study, computational time was not taken into account. But now, this must be taken into account since it is an on-line control determination.

\subsection{Real-time control}

The previous results show that, for this process, we are able to find optimal temperature and extent of cure corresponding to the optimal control. In order to take into account the on-line control problems (computational time limitation, modelling uncertainties and some disturbances), we adopt the internal linearized model control approach like in [4]. We just recall here the main ideas: the first one is to assume that the previous system $\left(S_{0}\right)$ is not really optimal but is not so far from the real-time optimal system. So, this variations can be described by a 
moving system $\left(\mathcal{S}_{T V L}\right)$ which is a time-varying linearized distributed parameter system :

$$
\left\{\begin{array}{l}
\Delta \omega_{t}(z, t)=A_{L}(t) \Delta \omega(z, t) \text { with } z \in \mathcal{D}, t>0 \\
B_{L_{1}}(t) \Delta \omega(z, t)+B_{L_{2}}(t) \Delta u(t)=0 \text { with } z \in \partial \mathcal{D}, t>0 \\
\Delta y_{m}(t)=C \Delta \omega(z, t) \text { with } z \in \mathcal{D} \cup \partial \mathcal{D}, t>0 \\
\Delta \omega(z, 0)=0
\end{array}\right.
$$

$A_{L}(t), B_{L_{1}}(t)$ and $B_{L_{2}}(t)$ are linear time-varying (LTV) operators obtained by the linearization of the nonlinear operators $A$ and $B$ around the standard operating point $\left(u_{0}(t), \omega_{0}(t)\right)$. The control problem is now to find the control variation $\Delta u(t)$, with $u(t)=u_{0}(t)+\Delta u(t)$ leading to the moved output $y_{m}(t)=y_{0}(t)+\Delta y_{m}(t)=$ $C\left(\omega_{0}(z, t)+\Delta \omega(z, t)\right)$, such that the process output $y_{p}(t)$ tracks the given optimal trajectory $y_{r e f}(t)$ : this timevarying control problem can be achieved using the IMC strategy:

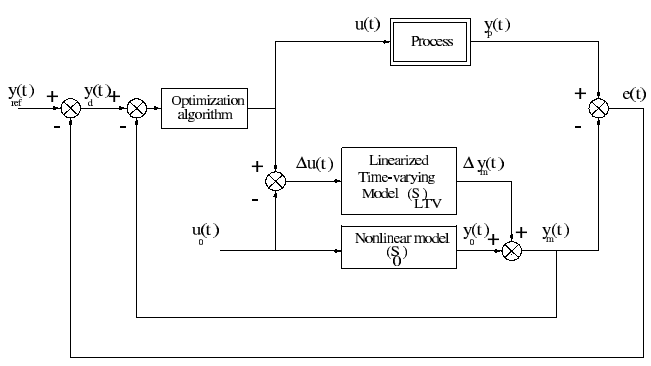

Figure 7: Internal linearized model control structure

\section{Conclusion}

In this work, we have presented a dual problem in the control of nonlinear parabolic distributed parameter system. It concerned the determination of the process control such that the operating time becomes the shortest possible and the classical induced tracking problem. For the former problem, we have used a model based approach with a predictive approach using nonlinear programming. The most important problem is to set constraints from the behaviour knowledge. Indeed, we still do not have an efficient model linking the autoclave temperature and the residual stresses induced during the cure cycle. Concerning the horizons tuning, a receding horizon of 1 seems to be sufficient like in the tracking problem. Concerning the prediction horizon, it has to be sufficiently important to take correctly into account of the constraints. Otherwise, the optimization problem is not well set and has no physical solution. For the tracking problem, we have introduced the Internal Model Control strategy with the same predictive approach. The internal model is the combination of a nonlinear model and its small perturbations model. The perspectives are a generalization study for the closed-loop stability.

\section{References}

[1] Banks S.P., "Controllability and optimal control of partial differential equations on compact manifolds", Int. J. Systems Sci., 15(5), 543-562, (1984).

[2] Barbu V., "Analysis and control of nonlinear infinite dimensional systems", Academic Press Inc, (1993).

[3] Courtial E., Touré Y., "Nonlinear Model Predictive control: constrained optimization and practical stability", International Symposium on Nonlinear Model Predictive Control, Ascona, Switzerland, Preprints p36., (1998).

[4] Dufour P., Touré Y., Laurent P., "A nonlinear distributed parameter process control: an internal linearized model control approach", CESA'98 IEEE IMACS Multiconference, Hammamet, 1, 134-138, (1998).

[5] Fletcher R., "Practical methods of optimization" John Wiley and Sons, (1987).

[6] Imai K., Kawahara M., "A study of water pollution control problem under inequality constraint on the control value", Proceedings of the International Conference on Nonlinear Mathematical Problems in Industry, 1, 237-251, (1993).

[7] LeClair S.R, Abrams F.L, "Qualitative process automation", Int. J. Integ. Manuf., 2, 205-211, (1988).

[8] Loos A.C, Nagendra S., "Optimization of the curing process of filament wound composites", Proc. of the 34th Int. SAMPE Symp., 1853-1867, (1989).

[9] Magri F., "Equivalence transformations for nonlinear evolution equations", Journal of Mathematical Physics, 18(7), 1405-1411, (1977).

[10] Pillai V., Beris A.N., Dhurjati P., "Heuristics guided optimization of a batch autoclave curing process", Computers Chem. Engng, 20(3), 275-294, (1996).

[11] Pillai V., Beris A.N., Dhurjati P., "Intelligent curing of thick composites using a knowledge-based system", Journal of Composite Materials, 31(1), 2251, (1997).

[12] Touré Y., Biston J., Gilles G., "Modeling of a distributed parameter process with a variable boundary : application to its control" Chemical Engineering Science, 49(1), 61-73, (1994).

[13] Touré Y., Josserand L., "An extension of IMC to boundary control of distributed parameter systems" IEEE International Conference on Systems, Man and Cybernetics-CCS Orlando Floride - USA, 3, 2426-2431, (1997). 